DOI 10.18699/PlantGen2019-159

\title{
Assessment genetic structure of Azerbaijan wild and cultivated barley genotypes by biochemical marker
}

\author{
Serpoush M. ${ }^{1 *}$, Salayeva S. ${ }^{2}$, Ojaghi J. ${ }^{1}$ \\ ${ }^{1}$ Department of Life Sciences, Khazar University, Baku, Azerbaijan \\ ${ }^{2}$ Department of Genetics and Evolutionary Theory, Baku State University, Baku, Azerbaijan \\ *e-mail:Moozhan.serpoush@khazar.org
}

A serious problem agriculture currently faces is the decreasing variability in genetic resources, which has resulted from the number of economically important crops being reduced and in addition, fewer varieties of these being used in place of many traditional varieties. Diversity in plant genetic resources provides opportunity for plant breeders to develop new and improved cultivars with desirable characteristics, which include both farmer-preferred traits and breeders preferred traits.

Extreme diversity of the soil and climatic conditions of Azerbaijan Republic support a very rich diversity of plant genetic resources. To preserve and use efficiently in the future the current genetic pool, it is necessary to evaluate the extent of this diversity by identification and distinction of Azerbaijan barley accessions, as well as the determination of genetic relationship between cultivated and wild relatives.

In order to investigate polymorphism resulting from monomeric prolamine and hordein, 49 wild and cultivated barley genotypes obtained from National Genbank of Azerbaijan Republic were analyzed. Monomeric prolamins and hordeins, which are part of storage proteins were identified by Acid-PAGE technique, using the Poperelya and Mujarinko's method. The difference in bands pattern can appear as an efficient tool in polymorphism. By analyzing of the hordeins, in each region 6 different patterns were observed separately. The average genetic diversity index for these proteins was calculated as PIC $=0.96$. In the analysis of the monomeric prolamins, 9, 7, 7, and 8 various patterns were observed for $\omega, \gamma, \beta$ and $\alpha$ areas, respectively. Also, the calculated average of the genetic diversity index for these proteins was the same of hordein seed storage $\mathrm{H}=0.95$.

Monomeric prolamines and hordein analyses were applied for the first time to obtain preliminary information on genetic profile of wild type barley samples in Azerbaijan. Our data suggests both markers are effective and reliable genetic markers for accurate assessment of genetic variation. Our analyses revealed high diversity between $H$. spontaneum and $H$. vulgare. Therefore, this information could be very useful for further management of Azerbaijan barley germplasm. 